\title{
Adolescent Pregnancy: Risk Factors, Outcome and Prevention
}

\author{
Fahmida Shirin Papri ${ }^{1 *}$ \\ Zubaida Khanam ${ }^{2}$ \\ Sarwat Ara \\ Morsheda Begum Panna ${ }^{3}$
}

'Department of Gynaecology \& Obstetrics Chittagong Medical College Hospital Chittagong, Bangladesh

${ }^{2}$ Department of Community Medicine Chittagong Medical College Hospital Chittagong, Bangladesh

${ }^{3}$ Department of Gynaecology \& Obstetrics Nagar Matri Sadan

Mamata, Lalkhan Bazar

Chittagong, Bangladesh.
*Correspondence to:

Dr. Fahmida Shirin Papri Assistant Professor

Department of Gynaecology \& Obstetrics

Chittagong Medical College

Chittagong, Bangladesh.

Mobile : +88 01711948384

Email :shirinfahmida44@yahoo.com

www.banglajol.info/index.php/CMOSHMCJ

\begin{abstract}
Adolescent pregnancy, which is detrimental to the health of mother and child, is a common public health problem worldwide. It is one of the key issues concerning reproductive health of women not only in developing countries but also in developed countries. There is growing awareness that early child bearing has multiple consequences in terms of maternal health, child health and over all well-being of the society. The purpose of the article is to review current trends and issues on adolescent pregnancy to update the practitioners. The readers are provided with more recent data on adolescent sexuality, child bearing as well as suggestions for addressing the challenges of teenage pregnancy.
\end{abstract}

Key words : Aolescent pregnancy; Risk factors; Outcome; Prevention.

\section{INTRODUCTION}

WHO's contribution to meet the Millenium Development Goals (MDGs) has given priority to the issues pertaining to the management of adolescent pregnancy. Adolescent is a period of the transition from childhood to adulthood. Pregnancy in a girl aged between 10-19 years is adolescent or teenage pregnancy ${ }^{1}$. By WHO definition adolescent or teen is defined as when aged 15-19 years². Adolescent birth rate is one of the expanded indicators of Millenium Development Goal in addition to the maternal mortality ratio, delivery by skilled birth attendant, contraceptive prevalence rate and antenatal care coverage ${ }^{1}$.

Teenage pregnancy is a global phenomenon. World wide, teenage pregnancy rates range from 143 per 1000 in some sub-Saharan African countries to 2.9 per 1000 in South Korea. Save the Children found that annually, 13 million children are born to women aged under 20 worldwide, more than $90 \%$ in developing countries. Complications of pregnancy and childbirth are the leading causes of mortality among women aged 15-19 in such areas. Highest risk of maternal death in young girls was shown in Africa, Afghanistan, Bangladesh, Guatemala, Haiti, Nepal, Nicaragua and Yemen ${ }^{3}$.

The pregnancy rate among teenagers in USA was 67.8 pregnancies per 1000 women aged 15-19 in 2008. The teenage birth rate in the United States is highest in the developed world and the teenage abortion rate is also high. In 2010 the birth rate in USA was 34.3 births per 1000 women aged 15-194.

The reported teenage pregnancy rate in South Asian countries like Bangladesh, Nepal and India are 35\%, 21\% and $21 \%$ respectively. Latest data suggests that teen pregnancy in India is high with 62 pregnant teens out of every 1000 women. The rate of early marriage and pregnancy has decreased sharply in Indonesia and Malayasia.In the industrialized Asian nations such as South Korea and Singapore, teenage birth rates are among the lowest in the world ${ }^{4}$. 
A major problem for the pregnant teen relates to her own body, and degree of both physical and emotional development achieved during the pubertal process. The incomplete development of genital tract and the musculoskeletal system of pregnant adolescents predispose them to worse overall obstetrical outcomes. There are high rates of spontaneous abortion, preterm delivery and low birth weight among adolescent girls as compared to older women aged 20$29^{[5]}$. Furthermore complications of pregnancy lead to twice as many deaths in adolescent compared to adult women. In addition to the increased maternal mortality rates, pregnancy can induce tremendous psychological stress on the adolescents, particularly with undesired pregnancies. The associated psychological and emotional burdens are economic responsibilities, adjustment in lifestyle and changes in family dynamic. Due to negative medical and social outcome, it is pertinent to launch interventions to avoid teenage pregnancies.Identification of the risk factors that influence the occurrence of teenage pregnancies is the basis on which effective preventive programmes should be developed.

\section{Risk Factors for Adolescent Pregnancies}

Factors increasing pregnancy among teenagers are numerous, and may vary between populations. Teenage pregnancy in developed countries usually occurs outside marriage, and in many communities and cultures carries a social stigma. In other countries and cultures particularly in developing world, teenage pregnancy is often within marriage and does not involve social stigma ${ }^{6}$. Residing in disorganized neighbourhoods and in a family with lower socioeconomic status, low educational attainment, living with a single parent, being a victim of sexual abuse, poor parent-child closeness, poor parental supervision or regulations of childrens' activities have been found to be factors elevating the risk of teenage pregnancy in USA. Socioeconomic disadvantage, disrupted family structure and limited education, risky sexual behaviour such as early sexual initiation, increasing number of partners and nonuse of contraceptives were the factors associated with teenage pregnancies in European Union Countries.

The risk factors identified for teenage pregnancy in South Asian countries like Bangladesh, India and Nepal include low socio- economic background, low educational attainment, disrupted family structure and poor sexual health practices ${ }^{4}$.

\section{Pregnancy Outcome of Teenage Pregnancy}

Adolescent pregnancy is associated with higher rates of morbidity and mortality of both the mother and infant.Adolescent sexual activity and pregnancy is concerned globally. A review on teenage pregnancy reported that $25 \%$ of all pregnancies in Sub-Saharan Africa and Asia end in induced abortion $^{3}$.
Nutrition and Weight in Pregnant Adolescent Nutrition is pivotal for fetal growth and is directly related to maternal anthropometry and placental volume. Low maternal weight and body mass index at conception or delivery, and poor weight gain during pregnancy have been associated with low birth weight, prematurity and maternal delivery complications. Teenage mothers are at nearly three times higher risk of having anaemia. Anaemia is of great obstetric concern as the condition is associated with several complications including low birth weight and increased risk of preterm delivery ${ }^{5}$.

\section{Preterm Birth}

Adolescent pregnancy are at increased risk for neonatal compclications as prematurity, low birth weight, Intrauterine Growth Retardation (IUGR) neonatal mortality and stillbirth. The maternal complications like preeclampsia,perineal tear and episiotomy are also common among adolescents. However, caesarean section, antepartum haemorrhage and postdated were not found significantly associated with adolescent pregnancy. Preterm labor has been associated with a number of factors including genital tract infection, extragenital chronic inflammation, stress and life-style factors which may be stronger determinants in pregnant adolescents than in adult pregnant women.In adolescents anatomical charachteristics of the cervix (Specially the short cervix) would favour preterm delivery ${ }^{5}$.

\section{Labor and Delivery}

Immaturity of the pelvic bones and of the birth canal may be a significant factor in obstetric risk in young adolescents. Evidence suggests that because of the relative immaturity of their physiological development, adolescents are more likely than older women to experience complications during delivery. Higher incidence of caesarean section, operative vaginal delivery(both vacuum and forceps extraction) and obstetric fistulas in adolescents, compared with that of older women, suggest an increased risk of prolnged and obstructed labour in adolescents. Some studies have shown that the risk of Caesarean section is increased in teenage pregnancy while some have shown the opposite ${ }^{6}$.

Furthermore children born to teens are more likely to have poorer long term educational, behavioraol and health outcomes than children born to older parents.

Treatment of Adolescent Pregnancy

Comprehensive prenatal care from the outset ensures a healthier baby. Smoking, alcohol use and drug abuse should be strongly discouraged in pregnant teens. Since pregnant teenagers carry a high risk of pregnancy, she must be cared in a hospital. Close antenatal check-up, advice on adequate diet, correction of anaemia, early detection of pre-eclampsia, advice on more rest to avoid premature births and good intranatal and postnatal care are all important. There should be adequate provision of access to effective contraceptive information and services for birth spacing, following delivery to discourage adolescent from becoming pregnant again. Adequate nutrition must be assured through both education and the availability of community resources ${ }^{7}$. 


\section{Prevention}

Many health educators have argued that comprehensive sex education would effectively reduce the number of teenage pregnancies. Interventions combining education and contraceptives appear to reduce unplanned teenage pregnancy, however no one intervention yet stands out as the most effective. Education could play a significant role in developing self-confidence increasing age at first sexual intercourse and delaying marriage. Compulsory sex education can help to empower the girls, which is the most effective strategy to prepare them for late marriage, planned and delayed pregnancy and better motherhood. The legal age of marriage for most South Asian countries is 18 years, however many girls marry before the age. Marriage Law in South Asia is unenforcible and might have limited effectiveness, it is also not in tune with cultural and social norms ${ }^{8}$.

A successful prevention program will include the following strategies (Modified from American academy of Pediatrics, Committee on Adolescence).

i. Adolescents should be encouraged to postpone early sexual activity. Abstinence counseling and information on and access to pregnancy prevention/termination, if they become sexually active, are an important.

ii. Physicians should be sensitive to issues relating to adolescent sexuality and be prepared to obtain a developmentally appropriate sexual history on all adolescent patients.

iii. It should be ensured that all adolescents who are sexually active have knowledge of and access to contraception. iv. Physicians should advocate for comprehensive medical and psychological support for all pregnant adolescents. Early and adequate prenatal care should be tailored to the medical,social,nutritional and educational needs of the adolescents and should include child care training as well.

v. Adolscent mothers should not receive early postpartum discharge so that clinicians can ensure that the mother is capable of caring for her child.

vi. The adolescent mother's partner and father of her child should be included in teenage pregnancy and parenting programs with access to education and vocational training, parenting skills classes, and contraceptive education.

We should serve as resources for pregnant teenagers and their infants, the teenager's family, and the father of the baby to ensure that optimal health care is obtained and appropriate support is provided ${ }^{1}$.

\section{CONCLUSION}

Pregnancy among adolescent is both a medical and a public health concern which may negatively impact the social and physical development of mother and affect reproductive quality within a given society.We should take step not only to improve the reproductive outcome but also decrease the incidence of teenage pregnancy by increasing public awareness, ensuring female education and enforcing marriage law. Teenage pregnancy needs to be tackled as a priority to ease the burden of socioeconomic and health problem.

DISCLOSURE

All the authors declared no competing interest. 


\section{REFERENCES}

1. G Dangal. An Update on Teenage Pregnancy.The internet Journal of Gynaecology and Obstetrics. 2004; 5(1).

2. United Nations, Millennium Development Goal : Updated 2012 http://unstatus.un.org/unsd/mdg/Service Details aspx?srid=761.

3. Liabsuetrakul T. Trends and Outcome of Teenage Pregnancy.Thai Journal of Obstetrics and Gynaecology. 2012; 20:162-164. .

4. Dulitha F, Nalika G,Upul S, De Silva C et al.Risk factors for teenage pregnancies in Srilanka: Perspective of a community based study.Health Science Journal. Suwal A. Obstetric and Perinatal Outcome of Teenage Pregnancy. J Nepal Health Res Counc. 2012;10(20):52-56

5. Perez-Lopez Faustino R,Chedraui, Kravitz A S, Salazar-Pousada D, Hidalgo L. Present Problems and Controversies Concerning Pregnant Adolescents.Open Access Journal of Contraception,Dovepress.2011;2:85-91.

6. Agudelo A C, Belizan J M, Lammers C. Maternal-perinatal morbidity and mortality associated with adolescent pregnancy in Latin America: Cross sectional study.American Journal of Obstetrics and Gynaecology. 2005;342-349.

6. S Sulaiman, S Othman, N Razali, J Hassan.Obstetric and Perinatal outcome in teenage pregnancies.SAJOG. 2013; 19(3):77-79.

7. Acharya DR, Bhattaria R, Poobalan A, Van Teijlingen ER, Chapman G.Factors associated with teenage pregnancy in South Asia: A systematic review. Health Science journal. 2010;4(1):3-14.

8. WHO.Towards adulthood:exploring the sexual and reproductive health of adolescent in South Asia .2003: World Health Organization, Geneva .Available from http/apps who int/reproductive health/publication/towards adulthood pdf, Accessed 3/05/09. 